\title{
Heavy-flavour production in fixed-target mode with $\mathrm{LHCb}$
}

\section{Felipe A. García Rosales*†}

Laboratoire Leprince-Ringuet, École Polytechnique, Institut Polytechnique de Paris Laboratoire de l'Accélérateur Linéaire, Université Paris-Sud, Université Paris-Saclay

E-mail: garciaellr.in2p3.fr

The LHCb detector has the unique capability to study collisions of the LHC beams on fixed targets. Internal gas targets of helium, neon and argon have been used so far to collect samples corresponding to integrated luminosities up to $0.1 \mathrm{pb}^{-1}$. An upgraded target, allowing a wider choice of target gas species and an increase in the gas density by up to two orders of magnitude, is planned to be installed for the LHC Run 3. Results on open and hidden charm production measurements in the case of proton beams against argon and helium targets are presented as well as fixed-target prospects. These measurements can provide crucial constraints on cold nuclear matter effects and nPDF at large $x$. In addition, production measurements of anti-protons and other light hadrons are of great interest for cosmic-ray physics.

European Physical Society Conference on High Energy Physics - EPS-HEP2019 -

10-17 July, 2019

Ghent, Belgium

\footnotetext{
* Speaker.

${ }^{\dagger}$ On behalf of the LHCb collaboration.
} 


\section{Introduction}

The LHCb detector is one of the main experiments of the Large Hadron Collider (LHC) [1]. It is a single-arm spectrometer in the forward direction covering an angle between 10 and $250 \mathrm{mrad}$ around the beam axis, which expressed in terms of the pseudo-rapidity, defined as:

$$
\eta=-\ln \tan \left(\frac{\theta}{2}\right)
$$

is equivalent to covering the range $2<\eta<5$. This particular geometry is designed for the study of particles containing $b$ or $c$ quarks.

Information coming from several sub-detectors allows to reconstruct very precisely the charged tracks and to identify them accurately. For the particle identification process there are dedicated muon stations, two Ring Imaging Cherenkov (RICH) detectors, which are a feature unique to LHCb, and a complex calorimeter system, made up of a Scintillator Pad Detector (SPD) to distinguish charged particles from neutral ones, a Pre-Shower (PS) detector to distinguish photons and electrons from hadrons, and Electromagnetic and Hadronic calorimeters (Ecal and Hcal) for energy measurement purposes. All of these detectors give LHCb an excellent Particle Identification (PID) capability.

For the charged track reconstruction, the detector relies on a high precision tracking system consisting on the Vertex Locator (VELO), located in the close vicinity of the interaction point of the beams and thus allowing to reconstruct very precisely the primary vertices and to differentiate them from the secondary ones, the magnet generating a magnetic field of 4 Tesla to bend the tracks, and other tracking stations placed before and after the magnet which allow to determine the topology of the different types of tracks [2].

LHCb has a System for Measuring the Overlap with Gas (SMOG) [3] which gives the unique possibility to inject gas into the interaction region, that is, in the tank that contains the VELO. SMOG's original purpose was to more precisely estimate the luminosity by allowing to make a precise profile of the beam, but it has also opened the possibility to use this gas as a fixed-target for the LHC beams, thus increasing LHCb capabilities by turning it, in addition, into a fixed-target experiment.

\section{Fixed-target operation}

Since 2015 the LHCb detector has been able to record data in a fixed-target configuration thanks to the SMOG set up. A schematic view of the collision scenario can be seen in the figure 1 . This type of collision is a unique feature within the LHC experiments.

The gas species that can be used are limited to noble gases, due to their low chemical potential, and so far the ones that have been used are helium, neon, and argon. A pictorial summary of the different scenarios can be seen in the figure 2. Depending on the beam energy used the centreof-mass energy ranges from $\sqrt{s_{N N}}=68.8-110 \mathrm{GeV}$, covering the energy gap between the CERN SPS $(\sim 20 \mathrm{GeV})$ and the BNL RHIC $(\sim 200 \mathrm{GeV})$. In fixed-target mode the centre-of-mass rapidity covered by the detector is shifted towards the backward region. At $\sqrt{s_{N N}}=110 \mathrm{GeV}$, the rapidity is shifted as $y^{*}=y_{l a b}-4.77$ and thus the range covered by LHCb is $-2.77<y^{*}<0.23$ allowing 
to probe the high Bjorken- $x$ region in the target nucleon. Therefore the SMOG, as a fixed-target programme, offers many new and unique possibilities for physics studies.

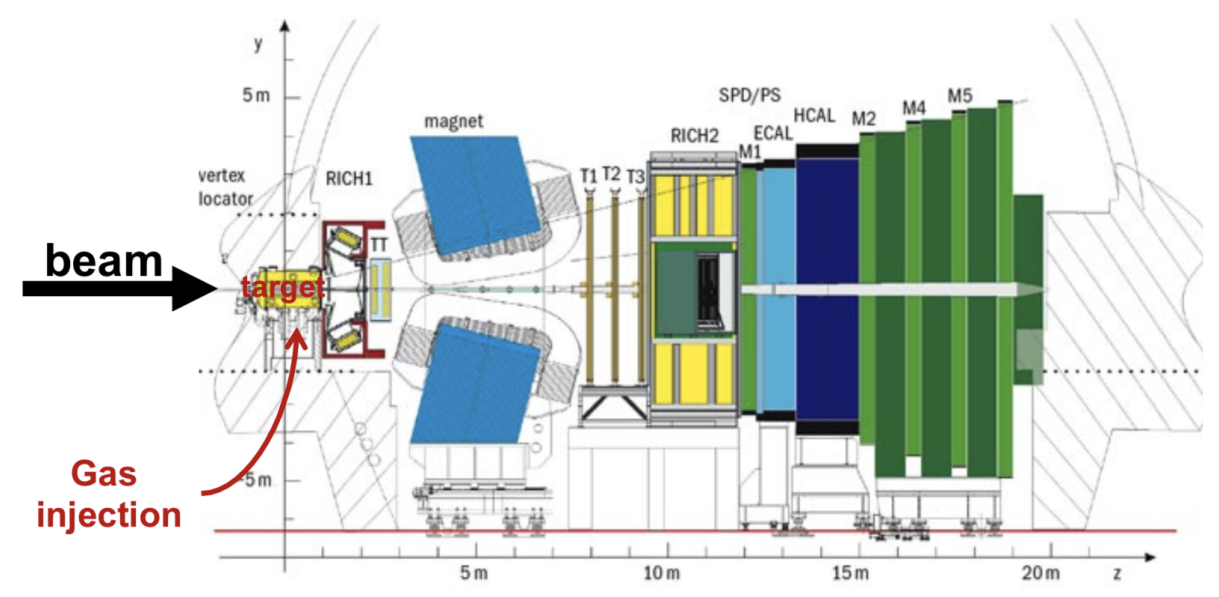

Figure 1: Schematic view of the LHCb detector, showing the corresponding sub-detectors. Also, the region where gas is injected as a target inside the VELO is pointed out.

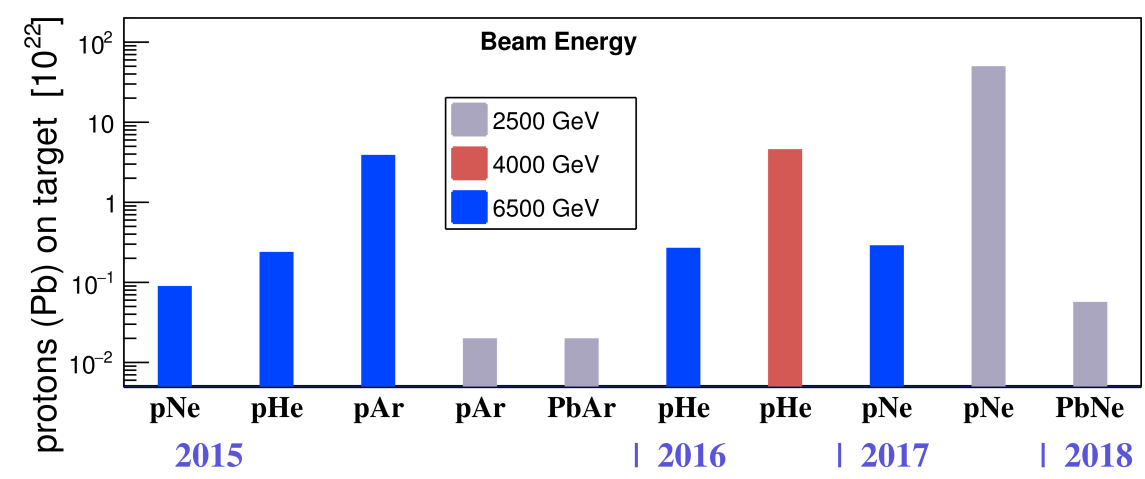

Figure 2: Different fixed-target collision systems for which data has been recorded by LHCb, with the respective beam energy used and estimated projectiles (protons or lead nuclei) on target.

\section{Charm production in $p$-A collisions}

The data analysed for the measurements presented here, correspond to proton beams colliding with gaseous targets of argon and helium at centre-of-mass energies of $110.4 \mathrm{GeV}$ and $86.6 \mathrm{GeV}$ respectively [4].

The samples have been collected under some specific conditions, only filled bunches from the beam going towards the detector cross empty bunches going in the reverse direction. The reconstructed vertex for events containing $J / \psi$ and $D^{0}$ candidates is required to be within the region $-200<z_{P V}<200 \mathrm{~mm}$, to exploit the high reconstruction efficiencies in the VELO. Events with activity in the backward region are vetoed in order to get rid of ghost $p p$ collisions. 
The $J / \psi$ and $D^{0}$ mass distributions extracted after the offline selection process can be seen in the figure 3. The $J / \psi$ signal is described by a crystal ball function and the $D^{0}$ signal is fit by the sum of two Gaussian functions. The background in both cases is described by an exponential function.

The luminosity information was only available for $p \mathrm{He}$, and was determined from the yield of electrons elastically scattering off the target helium atoms to be $\mathscr{L}_{p \mathrm{He}}=7.58 \pm 0.47 \mathrm{nb}^{-1}$. The measured cross-sections, within $2<y<4.6$ and after corrections for the respectives branching fractions were, for $J / \psi$ and $D^{0}$ (including charge conjugate)

$$
\begin{gathered}
\sigma_{J / \psi}=652 \pm 33 \text { (stat) } \pm 42 \text { (syst) } \mathrm{nb} / \text { nucleon } \\
\sigma_{D^{0}}=80.8 \pm 2.4(\text { stat }) \pm 6.3 \text { (syst) } \mu \mathrm{b} / \text { nucleon }
\end{gathered}
$$

Extrapolating to the whole phase space, assuming forward-backward symmetry, the total cross-sections are

$$
\begin{gathered}
\sigma_{J / \psi}=1225.6 \pm 100.7 \mathrm{nb} / \text { nucleon } \\
\sigma_{D^{0}}=156.0 \pm 13.1 \mu \mathrm{b} / \text { nucleon }
\end{gathered}
$$

where the uncertainties have been added in quadrature.
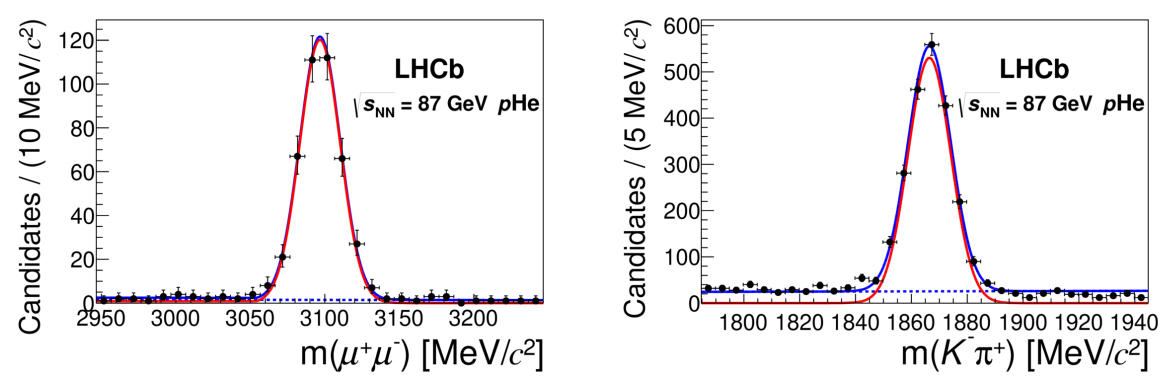

Figure 3: $J / \psi \rightarrow \mu^{+} \mu^{-}$(left) and $D^{0} \rightarrow K^{-} \pi^{+}$(right) mass distributions along with an unbinned maximum-likelihood fit on $p \mathrm{He}$ collisions at $\sqrt{s_{N N}}=86.6 \mathrm{GeV}$. The dashed blue line corresponds to the combinatorial background, the solid red line to the signal, and the solid blue line to the sum of the two.

The measured differential yields for $J / \psi$ and $D^{0}$ in $p$ Ar can be seen in figure 4 where they have been split into different rapidity $y^{*}$ and transverse momentum $p_{\mathrm{T}}$ bins. The first rapidity bin in the $D^{0}$ yield plot corresponds roughly to the Bjorken- $x$ region $x \in[0.17,0.37]$, where valence-like intrinsic charm should strongly contribute $[5,6]$. Since the data point falls below the theoretical prediction from HELAC-ONIA [7], which does not contain any intrinsic charm, there is no conclusive evidence of a strong intrinsic charm contribution to the nucleon.

\section{SMOG2: The upgraded fixed-target set up}

The current SMOG device allows the injection of noble gas in the beam pipe section crossing the VELO tank. To do this, two ion pumps of the VELO vacuum system are turned off and gas is injected reaching a pressure of the order of $10^{-7} \mathrm{mbar}$, which is about two orders of magnitude 

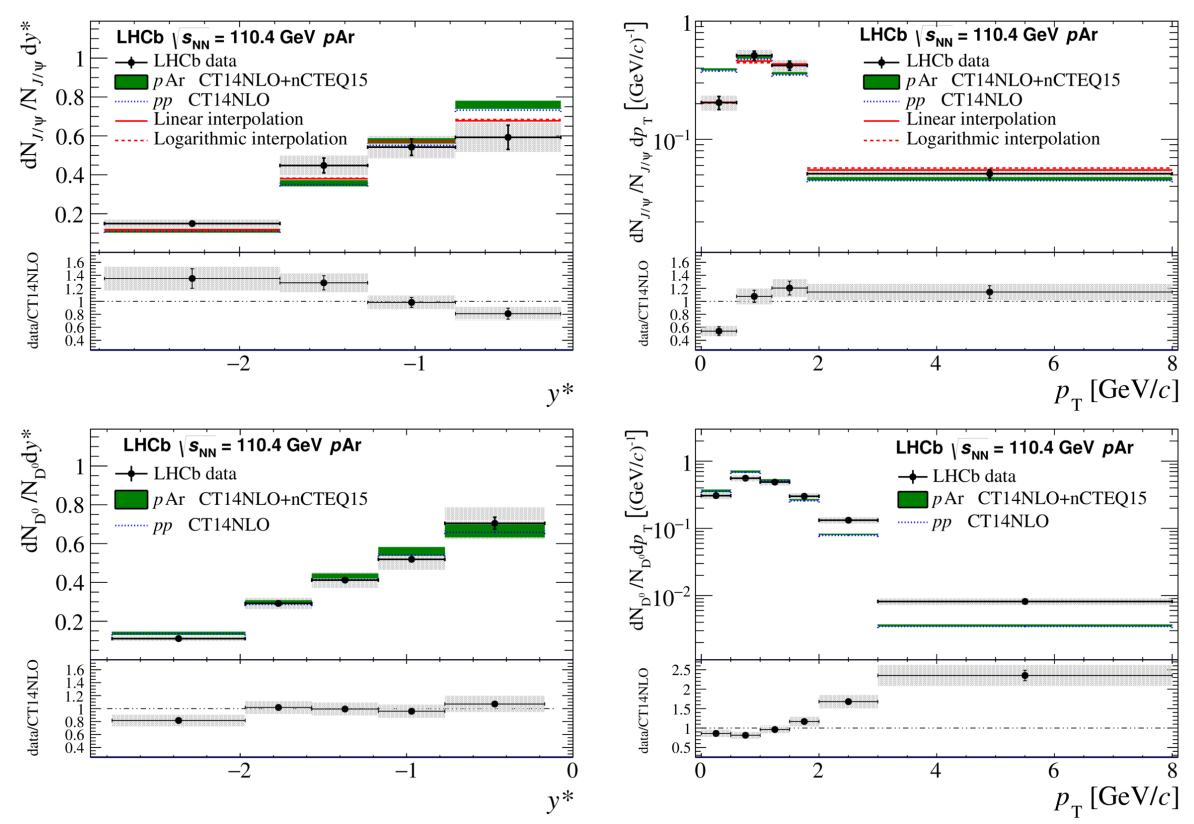

Figure 4: Differential yields for $J / \psi$ (top) and $D^{0}$ (bottom) as a function of centre-of-mass rapidity $y^{*}$ (left) and transverse momentum $p_{\mathrm{T}}$ (right) for $p$ Ar collisions. The data points mark the bin centres. The vertical black lines correspond to the quadratic sum of statistical and uncorrelated systematic uncertainties, and the grey boxes correspond to the correlated systematic uncertainties. Since for $p$ Ar luminosity information is not available, data are arbitrarily normalised to compare to theoretical predictions by HELAC-ONIA, consisting on phenomenological parametrisations to the present energy from data from experiments E789 [8], HERA-B [9] and PHENIX [10].

higher than the usual functioning pressure but still small enough to not significantly perturb the LHC operation. Then, the gas can flow into the beam pipes up to $\pm 20 \mathrm{~m}$ with respect to the nominal interaction point, where it is pumped out.

The upgraded system, named SMOG2, is to be installed by the end of 2019 and to be used for data taking in the Run 3 of the LHC [11]. It consists of a storage cell where the gas would be confined in a $20 \mathrm{~cm}$ long tube with $1 \mathrm{~cm}$ diametre. This storage cell would be attached to the upgraded VELO so that the actual target volume is located in the region $z \in[-500,-300] \mathrm{mm}$ with respect to the nominal interaction point, see figure 5. The storage cell is made up of two halves which follow the motion of the VELO detector, and thus can be retracted from their operating position when the beams are being injected, and they close around the beams when the beams are declared stable. The device can be seen in both open and closed position in the figure 6 as well as a more detailed view of one of the wings of the storage cell.

The design is made such that the VELO pumps do not need to be turned off. The gas density outside the cell is immediately suppressed, and the actual areal density of gas seen by the beam increases up to two orders of magnitude compared with SMOG for the same gas flow.

With this set up more gas species could be used, notably hydrogen $\left(\mathrm{H}_{2}\right)$ and deuterium $\left(\mathrm{D}_{2}\right)$ and heavier nobles gasses like $\mathrm{Kr}$ and $\mathrm{Xe}$. The device will be equipped as well with a more sophisticated gas feed system and monitoring that will allow to know with more precision the actual gas density and thus a more precise luminosity determination. 


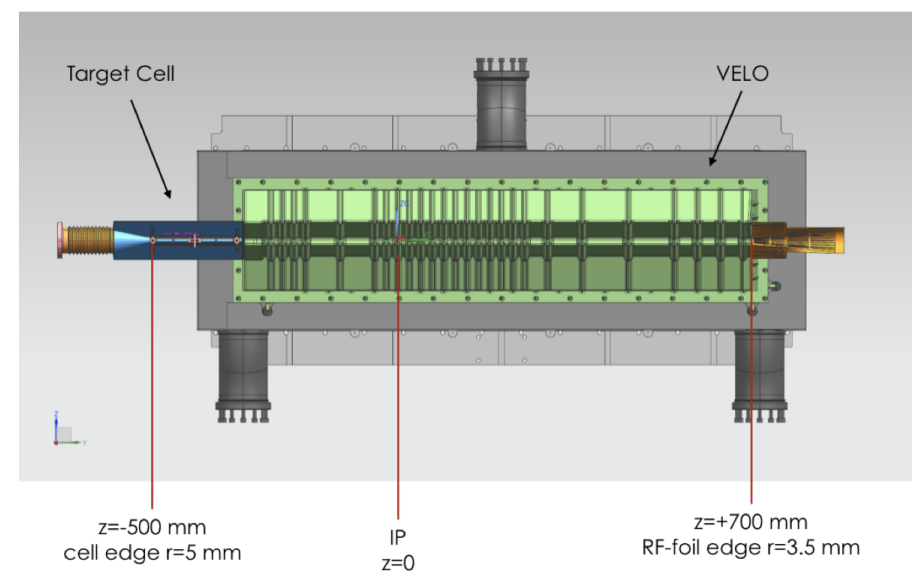

Figure 5: Layout of the SMOG2 storage cell with respect to the VELO. The nominal interaction point (IP) is shown. In the events of interest for fixed target physics the beam would cross from left to right.
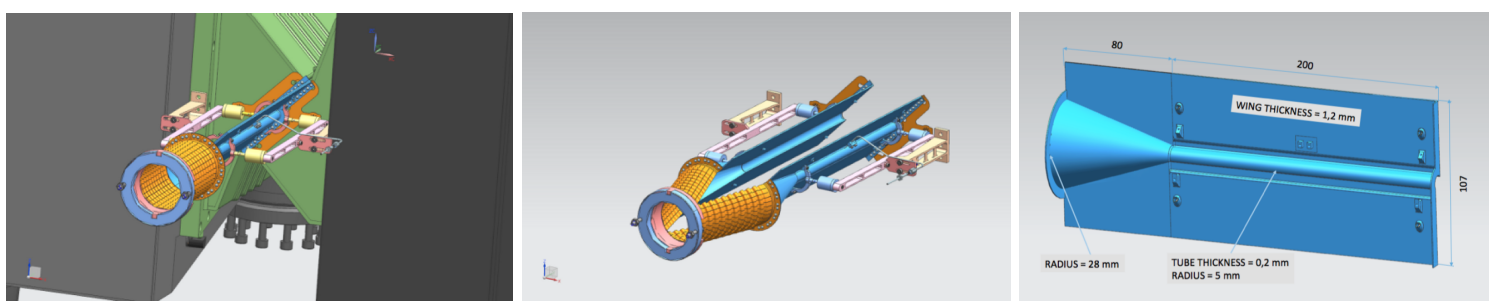

Figure 6: The SMOG2 storage cell in the closed (left) and open (middle) configurations. One of the wings of the SMOG2 storage cell (right).

A very important feature of the upgraded system is that by being displaced from the nominal beam interaction point, there would be no spacial overlap between the fixed-target operation and the beam-beam collisions. This means that background could be greatly reduced and that in principle all circulating bunches could be used for fixed-target physics. The latter possibility is still under study since it needs to be verified that the beam-gas interactions produce a tolerable background on the beam-beam physics and vice versa.

The physics prospects for SMOG2 are rich [12] and could contribute to different topics, such as hadron physics, cosmic ray physics and to the betterment of the understanding of the nucleon structure. In hadron physics new measurements of charm production with increased statistics, and the inclusion of other excited charmonia states, would allow to study with greater precision the suppression via colour screening expected with the formation of a Quark Gluon Plasma (QGP). In cosmic ray physics the knowledge of the content of antimatter in their composition is fundamental to use them as a way of indirectly probing the exotic antimatter production sources such as dark matter annihilation. The experiments PAMELA and AMS have improved substantially our knowledge in this topic, but there is still some tension with the predicted values for $\bar{p}$ fluxes due to known processes, mainly the interaction between primary cosmic rays and the interstellar gas, composed essentially of hydrogen and helium, see figure 7. In the $10-100 \mathrm{GeV}$ region the cross-section uncertainty dominates the total uncertainty, and since SMOG2 could replicate the collisions with hydrogen and helium it could provide an important input, reducing the uncertainty coming from 
the $\bar{p}$ cross-section. Finally for the nucleon structure, SMOG2 would allow to continue to probe the quark and gluon PDFs at high Bjorken- $x$ with increased statistical power, where experimental data is scarce, see figure 8 .

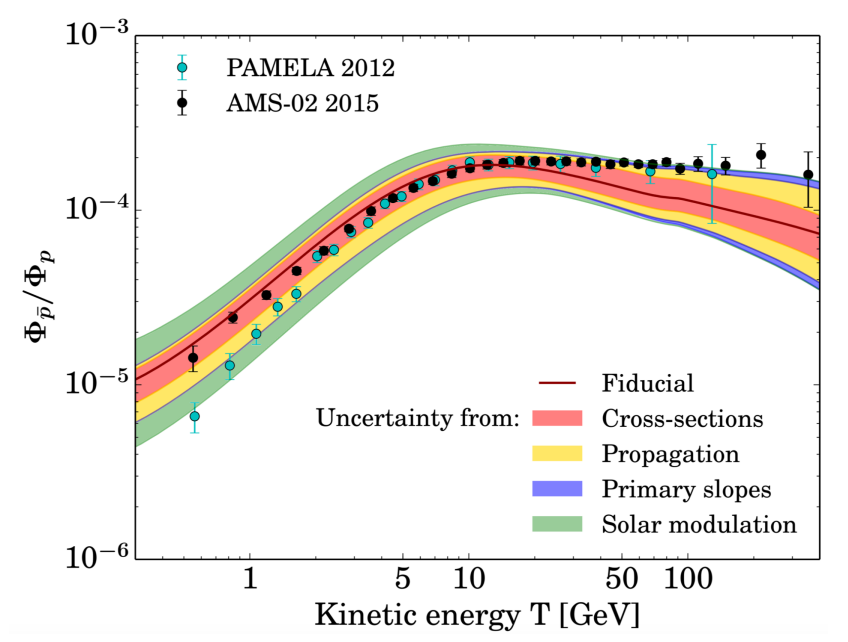

Figure 7: PAMELA and AMS data for the secondary $\bar{p} / p$ ratio, along with the predicted value and corresponding uncertainties [13].

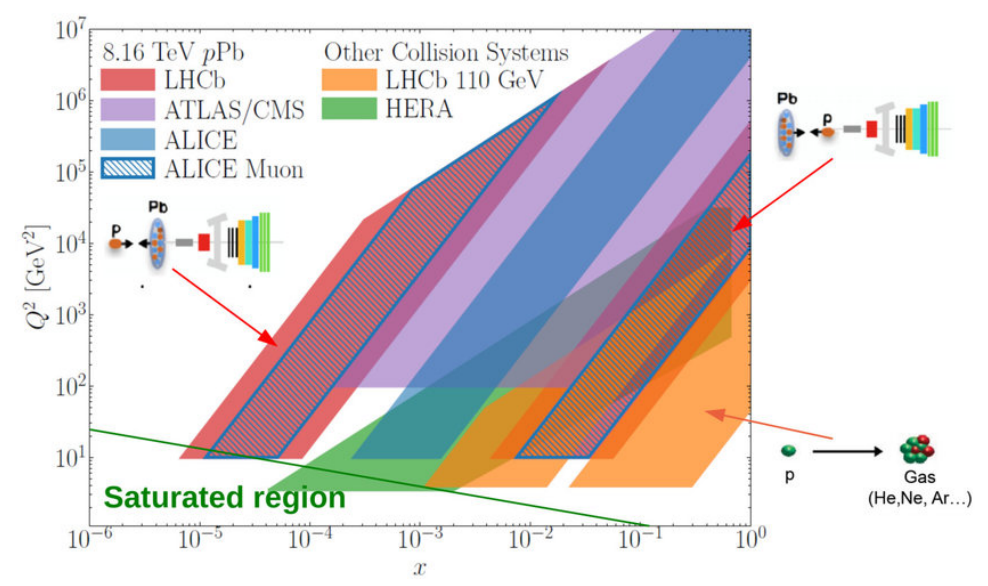

Figure 8: Accessible regions in the $\left(x, Q^{2}\right)$ plane for different experiments. LHCb is shown both in collider and fixed-target mode [14].

\section{Conclusions}

hsv Results on the measurement of hidden and open charm states were reported on $p \mathrm{He}$ and $p$ Ar collisions, where no evidence for strong intrinsic charm has been seen. There is still data to be analysed from Run 2, including proton-neon and lead-neon collisions at $\sqrt{s_{N N}}=69 \mathrm{GeV}$, which is an ongoing endeavour. The upgrade for SMOG, namely SMOG2 promises to drastically improve the fixed-target programme of $\mathrm{LHCb}$, with an increased luminosity, more versatile running conditions, and broader possibilities in the choice of a target, making it possible to expand significantly the physics reach of $\mathrm{LHCb}$. 


\section{References}

[1] LHCb collaboration, A. A. Alves, Jr. et al., The LHCb Detector at the LHC, JINST 3 (2008) S08005.

[2] LHCb collaboration, R. Aaij et al., LHCb Detector Performance, Int. J. of Mod. Phys A30 (2015) 1530022.

[3] LHCb collaboration, R. Aaij et al., Precision luminosity measurements at LHCb, JINST 9 (2014) P12005.

[4] LHCb collaboration, R. Aaij et al., First Measurement of Charm Production in its Fixed-Target Configuration at the LHC, Phys. Rev. Lett. 122 (2014) 132002.

[5] J. Pumplin, H. L. Lai, and W. K. Tung, Charm parton content of the nucleon, Phys. Rev. D 75 (2007) 054029.

[6] S. Dulat, T.-J. Hou, J. Gao, J. Huston, J. Pumplin, C. Schmidt, D. Stump, and C.-P. Yuan, Intrinsic charm parton distribution functions from CTEQ-TEA global analysis, Phys. Rev. D 89 (2014) 073004.

[7] J.-P. Lansberg, H.-S. Shao, Towards an automated tool to evaluate the impact of the nuclear modification of the gluon density on quarkonium, $D$ and $B$ meson production in proton-nucleus collisions, Eur. Phys. J. C 77:1 (2017).

[8] M. H. Schub et al., Measurement of J/ $\psi$ and $\psi^{\prime}$ production in $800 \mathrm{GeV} / \mathrm{c}$ proton-gold collisions, Phys. Rev. D 52 (1995) 1307.

[9] The HERA-B Collaboration, I. Abt et al, Kinematic distributions and nuclear effects of $J / \psi$ production in 920 GeV fixed-target proton-nucleus collisions, Eur. Phys. J. C 60525 (2009).

[10] PHENIX collaboration, A. Adare et al., $J / \psi$ production versus transverse momentum and rapidity in $p+p$ collisions at $\sqrt{s}=200$ GeV, Phys. Rev. Lett. 98 (2007) 232002.

[11] LHCb Collaboration, CERN (Meyrin), LHCb SMOG Upgrade, CERN-LHCC-2019-005. LHCB-TDR-020.

[12] A. Bursche et al., Physics opportunities with the fixed-target program of the LHCb experiment using an unpolarized gas target, LHCb-PUB-2018-015. CERN-LHCb-PUB-2018-015.

[13] G. Giesen et al., AMS-02 antiprotons, at last! Secondary astrophysical component and immediate implications for Dark Matter, JCAP 09 (2015) 023.

[14] G. Graziani, on behalf of the LHCb collaboration, Results on heavy ion physics at LHCb, Journal of Physics: Conf. Series 1271 (2019) 012008. 